\section{Experiencia del Comité de Ética de Investigación en Seres Humanos de la Facultad de Medicina de la Universidad de Chile y los desafíos que impone la nueva legislación chilena en la investigación médica}

\author{
MANUEL OYARZÚN G. ., MARÍA EUGENIA PINTO C.**, \\ GINA G. RAINERI B.***a, HUGO AMIGO ${ }^{\text {b }}$, LUCÍA CIFUENTES O., \\ MARÍA JULIETA GONZÁLEZc ${ }^{\mathrm{c}}$, NINA HORWITZ C. ${ }^{\mathrm{d}}$, \\ CLAUDIA MARSHALL F. ${ }^{\mathrm{e}}$, GRICEL ORELLANA V. ${ }^{\mathrm{f}}$
}

\section{Experience of a research Ethics Committee and the challenges of the new Chilean legislation on research in human beings}

The welfare of research participants must be guaranteed by international ethical standards. This article communicates the procedures of the Research Ethics Committee of the School of Medicine, University of Chile (CEISH). The new Chilean legislation on research in human beings is also discussed. Law 20.120: "On scientific research in human beings, its genome and forbidding human cloning" establishes the ethical principles that must be accomplished in every research involving human beings. Article 28 of the Law 20.584 "Regulation of the rights and duties of health care users", forbids the participation of handicapped people who cannot express their will in scientific research. Article 13 states that people not related directly with patient care cannot have access to his clinical records (with the exception of people with notarial authorization by the patient). CEISH proposes that, in case of people with intellectual deficiency, the decision to approve a scientific research should be analyzed on an individual basis. If the person is capable of expressing his or her will or has stated his or her consent beforehand, the research can be authorized. If the person cannot express his or hev will, the scientific research cannot take place. In prospective studies, a consent from the patient and an authorization of the health authority should be required to access clinical records. In retrospective studies, consent should be obtained from the patient when personal information is going to be used. If the information is nameless, the consent can be disregarded.

(Rev Med Chile 2014; 142: 889-895)

Key words: Ethics Committees, research; Ethics, research; Professional staff committees.

\author{
Comité de Ética de Investigación \\ en Seres Humanos: CEISH, \\ Facultad de Medicina, \\ Universidad de Chile. \\ *Presidente del Comité. \\ "Vicepresidente del Comité. \\ ***Secretaria del Comité. \\ aEnfermera-matrona y abogado. \\ Magíster en Bioética.

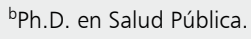 \\ ' Magister en Biología Celular. \\ ¿Socióloga y Magíster en Bioética. \\ 'Magíster en educación. \\ ${ }^{\mathrm{f}}$ Psiquiatra y Ph.D. en Ciencias \\ Biomédicas.
}

Manuscrito por invitación de los editores, sometido a revisión externa.

Conflictos de intereses: ninguno que declarar.

Recibido el 15 de abril de 2014, aceptado el 23 de junio de 2014.

Correspondencia a:

Dr. Manuel Oyarzún G.

Programa de Fisiopatología, ICBM, Facultad de Medicina,

Universidad de Chile. Avda.

Salvador 486.

Código Postal: 7500922

Correo 9. Providencia.

Teléfonos: 23419179-22741560

Fax: 22741628

moyarzun@med.uchile.cl

\section{L}

a investigación biomédica con seres humanos puede significar un beneficio para los Lacientes y para el desarrollo del país. La trayectoria e impacto de la investigación realizada en la Facultad de Medicina de la Universidad de Chile es una comprobación de esta relación beneficiosa ${ }^{1,2}$.

La investigación científica con participación de seres humanos proporciona valiosa información que de otra forma no podría obtenerse y se justifica 
éticamente por la expectativa de descubrir nuevas formas de favorecer la salud y el bienestar físico, psicológico y social de las personas. Sin embargo, este proceso no está exento de riesgos. Por lo mismo, los requisitos propuestos para que una investigación sea éticamente aceptable, están orientados a proteger y respetar a los sujetos de investigación que contribuyen al desarrollo del conocimiento ${ }^{3}$. Toda investigación en personas conlleva riesgos de daño físico o moral o de abuso con sus datos privados, de allí la necesidad de una regulación ética de la investigación ${ }^{4}$. Las regulaciones bioéticas han producido un impacto positivo en diversos aspectos de la investigación biomédica ${ }^{5}$. Para que una investigación sea ética, es crucial que los investigadores se preocupen por el bienestar de los voluntarios. Según Beecher, la garantía más fiable es que el proyecto tenga investigadores con buen criterio, informados y compasivos ${ }^{6}$.

El bienestar de quienes participan en investigación debe estar garantizado mediante estándares éticos compartidos internacionalmente. Reflejo de ellos es el proceso de "consentimiento informado", que resguarda la autonomía de los sujetos que participan en investigación ${ }^{4,5,7}$.

El objetivo del presente artículo es compartir la experiencia y modalidad de funcionamiento del Comité de Ética de Investigación en Seres Humanos de la Facultad de Medicina de la Universidad de Chile y discutir los alcances de la nueva legislación chilena en la investigación científica en seres humanos.

\section{Experiencia y funcionamiento del Comité de Ética en investigación en seres humanos (CEISH) de la Facultad de Medicina, Universidad de Chile}

Este comité se creó para responder a la creciente necesidad de regulación y supervisión ética de la investigación científica en sujetos humanos, que realizan académicos y alumnos de pregrado, de postgrado y de postítulo en la Facultad de Medicina de la Universidad de Chile. El trabajo del CEISH tiene por objetivo primordial $^{3}$ la protección de las personas que participan voluntariamente en un proyecto de investigación científica (Tabla 1).

El CEISH está constituido por nueve miembros, ocho de ellos académicos de la Facultad y el noveno es una educadora representante de la comunidad. De los académicos, cuatro son médicos especialistas (Genética, Microbiología, Neumología, Neuropsiquiatría), dos son profesionales (una abogada y enfermera-matrona, y una socióloga) con postgrado en Bioética. Completan sus integrantes una Bióloga Celular y un especialista en Salud Pública.

El comité se reúne quincenalmente. Los acuerdos se toman por consenso y sus resoluciones tienen carácter vinculante, es decir, las resoluciones del comité deben ser aplicadas por el comité, por los investigadores del proyecto y en la resolución del mismo proyecto ante otros comités de ética. Estas resoluciones van conformando los criterios a aplicar por el comité para asegurar el bienestar de quienes participan en investigación. En la revisión de proyectos de investigación, el CEISH aplica un procedimiento sistematizado (Tabla 2). La pauta de revisión contiene 17 puntos que evalúan la validez del estudio y su metodología, eventuales riesgos de los voluntarios, cumplimiento de normas de buena práctica clínica, proceso de consentimiento informado, competencia de los investigadores y financiamiento del proyecto.

En los últimos 4 años el CEISH ha analizado un número creciente de proyectos de investigación de los académicos y estudiantes de pre y post-grado: desde 89 proyectos (año 2010) a 165 proyectos (año 2013). El porcentaje de aprobación fue de $84 \%$ en estos 4 años; $16 \%$ restante incluye proyectos rechazados $(2 \%)$, proyectos retirados por los propios investigadores y aquellos cuyos investigadores no respondieron a las observaciones del comité, en su gran mayoría referentes al proceso del consentimiento informado. Las causas

\section{Tabla 1. Objetivos del Comité de Ética de Investigación en Seres Humanos. Facultad de Medicina, Universidad de Chile ${ }^{3}$}

1. Supervisar la debida protección de sujetos involucrados en la investigación científica, biomédica y psicosocial, realizada por académicos y estudiantes de la institución y el resguardo conforme a los principios bioéticos y normas internacionales de los estudios realizados

2. Brindar apoyo a los investigadores en los aspectos éticos de sus proyectos

3. Promover la formación en ética de la investigación en los investigadores y estudiantes de las unidades académicas y en los propios miembros del Comité 
Nueva legislación chilena en la investigación médica - M. Oyarzún et al

Tabla 2. Etapas del procedimiento en la revisión de proyectos por el CEISH

- Asignación del proyecto a uno o dos miembros del CEISH

- Revisión según pauta y elaboración de informe de revisión

- Discusión y resolución en sesión quincenal, cuyas opciones son:

a) Otorgar "Acta de aprobación" fundamentada del proyecto

b) Rechazar el proyecto con fundamentación.

c) Enviar carta al investigador responsable (IR) solicitando responder a las observaciones del Comité*

* La respuesta del IR es analizada por el CEISH en la siguiente sesión

- Si persisten dudas y problemas se cita al IR a sesión del CEISH para aclarar y dar solución

- Emisión del acta de aprobación cuando se haya dado respuesta a los reparos del CEISH

CEISH: Comité de Ética de Investigación en seres humanos.

de rechazo de proyectos por el comité fueron: relación riesgo/beneficio inadecuada, el proyecto ya se había ejecutado y metodología y proceso de consentimiento informado inapropiados.

\section{Legislación vigente relacionada con la} investigación biomédica en seres humanos ${ }^{8,9}$

Las leyes 20.120 y 20.584 están directamente relacionadas con la investigación científica en seres humanos.

La ley 20.120 plantea los puntos señalados en la Tabla $3^{8}$, en que se especifica que la aprobación del Comité Ético-Científico debe ser previa a la autorización del director del establecimiento. Por otra parte, esta ley y su reglamento declaran que la investigación científica biomédica es indispensable para el progreso de las ciencias de la salud y, por ende, para el bienestar de la sociedad ${ }^{8,10}$. El reglamento, en su artículo 12, al referirse al proceso de consentimiento informado, señala que debe ser otorgado por la persona en quien se realizará la investigación o por su representante legal y en un inciso siguiente en relación a la investigación científica con sujetos con discapacidad psíquica o intelectual, se deberá tener en cuenta lo señalado en la ley $\mathrm{N}^{\circ} 20.584$ y su reglamentación pertinente, cuando corresponda.

\section{Tabla 3. Ley $\mathbf{2 0 . 1 2 0 ~ " S o b r e ~ l a ~ I n v e s t i g a c i o ́ n ~}$ científica en el ser humano, su genoma, y prohíbe la clonación humana" (Inicio de vigencia: 22.09. 2006)}

- Toda investigación física o psíquica en seres humanos debe ser realizada por profesionales idóneos justificando objetivos y metodología

- Prohíbe realizarla cuando hay riesgo de muerte o lesión grave y duradera

- Debe realizarse con la aprobación de un Comité ÉticoCientífico y autorización del director del establecimiento en que será efectuada

- Debe contar con el consentimiento previo, personal, libre e informado por escrito del voluntario o de su representante legal

Tabla 4. Artículos de la Ley 20.584 que plantean limitaciones en las investigaciones científicas en seres humanos

Art 28. Prohíbe la participación en investigaciones científicas a personas con discapacidad psíquica o intelectual que no puedan expresar su voluntad

Art 13. Dispone que los "terceros" (personas) que no estén relacionados directamente con la atención de salud de la persona no tendrán acceso a la respectiva ficha clínica, considerando las siguientes excepciones:

a) Titular de la ficha, su representante legal o sus herederos*

b) Un "tercero" autorizado ante notario por el titular

c) Los tribunales de justicia**.

d) Los fiscales del Ministerio Público y abogados, previa autorización del juez competente***

* Los herederos son excepción sólo tras el fallecimiento del paciente. ** Los tribunales están autorizados sólo si la información tiene relación con la causa que estuvieren conociendo. ${ }^{* * *}$ Limitado a lo vinculado directamente con la causa en investigación o defensa.

Por su parte la ley 20.584 sobre derechos y deberes de los pacientes plantea dos artículos (Tabla 4) que representan un potencial impacto negativo en la investigación científica ${ }^{4,9}$.

\section{Artículo 28 de la ley 20.584}

Establece que "Ninguna persona con discapacidad psíquica o intelectual que no pueda expresar su voluntad podrá participar en una investigación científica". "En los casos en que se realice investi- 
gación científica con participación de personas con discapacidad psíquica o intelectual que tengan la capacidad de manifestar su voluntad y que hayan dado consentimiento informado, además de la evaluación ético científica que corresponda, será necesaria la autorización de la Autoridad Sanitaria competente, además de la manifestación de voluntad expresa de participar tanto de parte del paciente como de su representante legal".

El artículo 28 imposibilita efectuar investigación científica en personas con discapacidad psíquica que no puedan manifestar su voluntad. Lo cual constituye una seria limitación para realizar investigación en personas con daño neurológico, situación que en el pasado se superaba con el consentimiento informado otorgado por un representante legal del paciente ${ }^{4,11}$. Esta situación no sólo preocupa a los Comités de Ética que han debido rechazar proyectos de investigación según el artículo 28 y a los investigadores afectados, sino que, paradójicamente, a los propios pacientes, quienes a través de sus asociaciones o agrupaciones de enfermos y sus familiares (portadores de enfermedad de Alzheimer, síndrome de Down o enfermedad de Huntington) han manifestado públicamente su rechazo a esta medida legal, que restringe su participación en investigación cuando están en etapa avanzada de su enfermedad ${ }^{4}$.

\section{Planteamiento del CEISH sobre la aplicación del artículo 28}

El CEISH considera que se deben tomar decisiones caso a caso y en base a tres pilares fundamentales:

1. La no discriminación de los pacientes con discapacidad, especialmente en consideración a la Constitución Política de la República y los tratados internacionales, normas dentro de nuestro ordenamiento jurídico de mayor jerarquía, debiendo las de menor jerarquía concordar con aquellas, conforme al ordenamiento jurídico vigente.

2. La fundamentación entregada por el investigador responsable de la necesidad de realizar investigación en este grupo de personas.

3. La evaluación ética del proyecto realizada por el CEISH, considerando los dos puntos anteriores.

En pacientes con discapacidad psíquica/intelectual se debe considerar además su capacidad para tomar decisiones y las pautas éticas internacionales de investigación biomédica en poblaciones vulnerables ${ }^{12}$, tomando los resguardos necesarios para evitar cualquier coacción, de manera que el voluntario tome libre e informadamente su decisión de participar.

En etapas más avanzadas de deterioro, lo prudente es no considerar a estos pacientes como sujetos de investigación, mientras no se modifique la ley $\mathrm{N}^{\circ}$ Ley $20.584^{9}$. El CEISH propone exceptuar de la aplicación de esta ley al paciente que haya manifestado directrices anticipadas en un documento, es decir, cuando el voluntario estaba con capacidad de tomar decisiones haya manifestado por escrito su decisión libre e informada de ser incluido en un protocolo de investigación.

En caso de pacientes en estado de coma, aunque sea producido por situaciones transitorias o agudas, un representante del paciente no puede autorizar su participación en investigación. Así lo confirman los principios éticos y tratados internacionales y el artículo 28 de la ley mencionada.

\section{Reflexiones del CEISH sobre el artículo 28}

La definición de discapacidad psíquica e intelectual que estableció el reglamento de la ley 20.584 en su art. 19 es muy amplia: "Se considerará persona con discapacidad psíquica o intelectual a aquella que, en forma asociada a una enfermedad mental, manifiesta un daño o limitación, temporal o permanente, en cuanto a su funcionamiento intelectual o adaptativo a la vida social y que, al interactuar con obstáculos del entorno, ven impedida o restringida su participación efectiva en igualdad de condiciones con las demás personas." (Decreto 38/2012 Minsal).

El diagnóstico de discapacidad física e intelectual es controversial y requiere certificación por especialistas. El Comité de Ética deberá determinar si la investigación puede ser realizada. Los criterios y grados de suficiencia deben considerar además la fundamentación de los investigadores sobre la legitimidad de investigar en pacientes discapacitados.

Los principios para que una investigación sea éticamente aceptable, si bien son universales, deben adaptarse según las condiciones sanitarias, económicas, culturales y tecnológicas en que se está llevando a efecto la investigación clínica ${ }^{5,6}$. Una postura restrictiva deriva en tres aspectos: primero en un perjuicio a los investigadores; segundo, afecta socialmente a los pacientes en su 
autonomía al impedir que puedan recibir un beneficio eventual que se genere desde la investigación; y tercero, se debe distinguir entre el "diagnóstico psiquiátrico" y la "discapacidad", pues ambos términos no son sinónimos ${ }^{13}$.

Se estima que 5 a $10 \%$ de la población general puede presentar limitaciones en su capacidad para decidir participar en un proyecto de investigación. Este porcentaje es superior en sujetos con enfermedades cerebrales. La valoración de la capacidad de los sujetos para tomar la decisión de participar o no en un estudio es necesaria si queremos garantizar la validez y la calidad del consentimiento informado en investigación. Así se respeta la autonomía del sujeto y se le puede proteger en el caso de que esta capacidad esté mermada ${ }^{13}$.

La evaluación de la capacidad de las personas para tomar decisiones resulta compleja e implica una gran responsabilidad, tanto ética como legal para los investigadores ${ }^{14}$. Se deben utilizar instrumentos estandarizados y validados en nuestro país, para evaluar las funciones cognitivas que orienten sobre la capacidad para tomar decisiones en pacientes neuropsiquiátricos. Una proporción de ellos presenta habilidades cognitivas dentro de límites normales o leve alteración, y por ende podrá comprender un consentimiento informado ${ }^{14}$. Considerando lo anterior, la decisión del CEISH requiere una opinión de experto y debe ser flexible y situada para cada caso en particular.

En relación a la "evaluación cognitiva de la capacidad de consentir", los avances en neurociencias han mejorado la comprensión de las enfermedades neuro-psiquiátricas.

Clínicamente, lo primero que se debe analizar en un paciente neuropsiquiátrico es la función cognitiva global, por ejemplo con WAIS (Wechsler Adult Intelligence Scale). Este test determina el cuociente intelectual (CI), el cual permite precisar si la persona presenta o no un retraso mental ${ }^{15}$. Luego se examinan con pruebas específicas las funciones cognitivas más discretas como atención, memoria y toma de decisiones. Finalmente, para precisar el grado de funcionalidad e independencia del individuo se aplican a él y a su cuidador encuestas que miden "actividades de la vida diaria": $\mathrm{AVD}^{16}$.

La discapacidad cognitiva debe ser analizada ética y jurídicamente, pues no realizar investigación en personas con discapacidad puede derivar en una discriminación negativa, viéndose afectado el principio de justicia y las garantías constitucio- nales en relación al acceso a las acciones de salud, pues se le negarían a estos pacientes los beneficios que trae consigo la investigación médica en las diferentes acciones de salud garantizadas. Por otra parte, realizar investigación de acuerdo a estándares éticos y metodológicos de mayor exigencia, permitiría que estos pacientes se beneficien de investigaciones que resulten de valor para sus patologías.

La no discriminación está consagrada en la Constitución y en la Convención Internacional de las Naciones Unidas sobre los derechos de las personas discapacitadas ${ }^{17}$. El propósito de esta Convención es "promover, proteger y asegurar el goce pleno y en condiciones de igualdad de todos los derechos humanos y libertades fundamentales por todas las personas con discapacidad, y promover el respeto de su dignidad inherente".

Las personas con discapacidad "incluyen a aquellas que tengan deficiencias físicas, mentales, intelectuales o sensoriales a largo plazo que, al interactuar con diversas barreras, puedan impedir su participación plena y efectiva en la sociedad, en igualdad de condiciones con las demás"17.

Se ha planteado que el espíritu de estas regulaciones éticas de la investigación científica en personas vulnerables tiende a asegurar una situación igualitaria respecto a los derechos sociales y su ejercicio público. Se ha buscado prevenir abusos y/o discriminaciones que puedan derivarse de una falsa concepción de beneficencia de la actividad científica, que pudiese resultar lesiva para la dignidad y los intereses de las personas, si se anteponen los fines del conocimiento a los de la propia vida humana que se pretende conocer ${ }^{18}$.

\section{Artículo 13 de la ley 20.538}

El artículo 13 establece que quienes no estén directamente relacionados con la atención de salud de la persona no tendrán acceso a la información contenida en la ficha clínica ${ }^{4,11}$. Entre las excepciones a este mandato del artículo 13 (Tabla 4) no se contempla el acceso a la información contenida en la ficha clínica para investigación clínica o epidemiológica. Por lo tanto, el titular de la ficha, o sea el paciente, debería otorgar una autorización notarial para tal fin. Esta limitación, si bien es relativa, podría crear en innumerables casos un bloqueo no superable para realizar investigación clínica y epidemiológica, tan indispensa- 
bles para auscultar la realidad clínica y sanitaria de nuestro país. Otra implicancia de esta disposición es la potencial limitación que provocará en la publicación de casos clínicos en revistas biomédicas, que son de gran utilidad para el progreso de las ciencias médicas. En este contexto legal, los comités editoriales de revistas nacionales podrían exigir para publicar un caso clínico, la autorización del Comité de Ética institucional además de las habituales medidas para proteger el anonimato del paciente, cuyo caso es presentado ${ }^{4}$.

Esta situación fue tratada por la circular A15/15, de 18 de abril de 2013, del Ministerio de Salud, titulada "Emisión de consentimiento de personas participantes de una investigación científica", la cual aclara la relación entre la ley 20.584 que "Regula los derechos y deberes que tienen las personas en relación con acciones vinculadas a su atención en salud"9 y la ley 20.120 "Sobre la investigación científica en el ser humano, su genoma $y$ prohíbe la clonación humana", explicando que por tratarse la ley 20.120 de una ley específica sobre una materia -investigación científica- sus disposiciones tienen preeminencia en estos casos sobre las de la ley 20.584 y, por ello, no se requiere que el consentimiento informado se otorgue ante notario, como lo establece la ley $\mathrm{N}^{\circ} 20.584$. Siguiendo el argumento de esta circular se puede inferir que para acceder a los antecedentes de la ficha clínica en un proyecto de investigación bastaría contar con un consentimiento informado por parte del sujeto de investigación, con lo cual se resolvería el problema. Sin embargo, se ha planteado que esto no sería suficiente, ya que habría dos tipos de consentimiento informado: uno para participar como sujeto de la investigación y otro para autorizar al investigador el acceso a la ficha clínica, ambos requieren que sean consentimientos expresos y el otorgamiento de uno no supone el otorgamiento del otro ${ }^{19}$.

\section{Planteamientos del CEISH en la aplicación del artículo 13}

El procedimiento que propone utilizar el CEISH para autorizar el uso de fichas clínicas en investigación es diferente si se trata de estudios prospectivos o retrospectivos.

En los estudios prospectivos se debe solicitar consentimiento por escrito, en un proceso en que participan: el eventual voluntario, el investigador responsable y el director de la institución o su de- legado (Ley Base Procedimientos Administrativos del Estado).

En los estudios retrospectivos si la información será usada en forma no anónima o personalizada, se debe realizar el proceso de consentimiento informado. En cambio, si la información será utilizada sobre datos previos en forma anónima (base de datos anónima, en que los datos identificadores están encriptados), no se requiere del proceso de consentimiento informado ya que el CEISH lo puede "dispensar", por lo que en tal caso no se requerirá contactar al titular del dato sensible. Para otorgar esta dispensa el CEISH debe considerar el cumplimiento de los siguientes criterios: a) confidencialidad de la declaración; b) asegurar el anonimato del paciente (encriptación de datos identificadores; c) responsabilidad/compromiso del investigador; d) valor y validez científica de la investigación; e) justificación social y científica de la investigación propuesta. En este mismo sentido se debería preconizar la elaboración de bases de datos codificados sobre problemas clínicos y de salud pública. En la Escuela de Salud Pública de nuestra institución se ha propuesto la creación de un "Centro Coordinador de Datos", diseñado para entregar soporte a los investigadores en el correcto manejo de la información de estudios clínicos y/o epidemiológicos en seres humanos, garantizando la confidencialidad, seguridad y calidad de los datos, con el debido resguardo ético que exige la práctica científica y la legislación chilena (Eric Tapia, comunicación personal).

Finalmente, debemos enfatizar que el nuevo marco legal no ha modificado el papel que desempeñaban los Comités de Ética de Investigación antes de la promulgación de la nueva legislación ${ }^{20}$. Más aun, se ha destacado que la normativa legal actual ratifica que la responsabilidad primordial de estos comités es proteger a los sujetos de investigación ${ }^{21}$.

Agradecimientos: A los Drs. Miguel O’Ryan y Gustavo Kaltwasser, por su valiosa participación en algunas de las discusiones sobre este tema en el CEISH.

\section{Referencias}

1. Goic A. Facultad de Medicina de la Universidad de Chile: 170 años al servicio del país. Rev Med Chile 2003; 131 (4): 355-8. 
2. Valdés G, Armas Merino R, Reyes H. Principales características de la investigación biomédica actual, en Chile. Rev Med Chile 2012; 140 (4): 484-96.

3. Reglamento Comité de Ética de Investigación en Seres Humanos. Facultad de Medicina, Universidad de Chile. Santiago, 13 de marzo, 2012.

4. Raineri GG, Oyarzún M. Potencial impacto de la nueva legislación en la investigación biomédica en Chile. Rev Chil Enf Respir 2013; 29 (1): 5-8 (Editorial).

5. Gaudlitz M. Reflexiones sobre los principios éticos en investigación biomédica en seres humanos. Rev Chil Enf Respir 2008; 24 (2): 138-42.

6. Beecher HK. Ethics and clinical research. N Engl J Med 1966; 274 (24): 1354-60.

7. Emanuel EJ, Wendler D, Grady C. What makes clinical research ethical? JAMA 2000; (20) 283: 2701-11.

8. Gobierno de Chile. Ley 20.120 Sobre la investigación científica en el ser humano, su genoma y prohíbe la clonación humana. 7 de septiembre de 2006.

9. Gobierno de Chile. Ley 20.584 Sobre derechos y deberes de los pacientes en relación con acciones vinculadas a su atención en salud. 13 de abril de 2012.

10. Subsecretaría de Salud Pública. Minsal. Reglamento 114. Aprueba Reglamento de la Ley 20.120.

11. O’Ryan M Investigación médica en Chile a la luz de la nueva legislación. Rev Chil Infectol 2013 (1): 7-9.

12. Pautas éticas internacionales para la investigación biomédica en seres humanos. CIOMS/OMS. Ginebra 2002. Edición en Lengua española. Disponible en: http:// www.ub.edu/rceue/archivos/Pautas_Eticas_Internac.pdf Fecha de la consulta: 5.06.2014.

13. Badcock JC, Dragović M, Waters FA, Jablensky A. Dimensions of intelligence in schizophrenia: evidence from patients with preserved, deteriorated and compromised intellect. J Psychiatr Res 2005; 39 (1): 11-9.

14. Baón-Pérez BS. "Adaptación y validación española de la entrevista MacArthur competency assessment tool for clinical research (MACCAT-CR) y de un cuestionario breve para evaluar la capacidad de las personas para consentir participar en investigación. Memoria para optar al grado de doctor. Facultad de Medicina, Universidad Complu- tense de Madrid. Madrid, 2013.

15. Harman-Smith YE, Mathias JL, Bowden SC, Rosenfeld JV, Bigler ED. Wechsler Adult Intelligence Scale-Third Edition profiles and their relationship to self-reported outcome following traumatic brain injury. J Clin Exp Neuropsychol 2013; 35 (8): 785-98.

16. Johnson N, Barion A, Rademaker A, Rehkemper G, Weintraub S. The Activities of Daily Living Questionnaire: a validation study in patients with dementia. Alzheimer Dis Assoc Disord 2004; 18 (4): 223-30.

17. Decreto Núm. 201.- Santiago, 25 de agosto de 2008, Ministerio Relaciones Exteriores "Promulga la Convención de las Naciones Unidas sobre los derechos de las personas con discapacidad y su protocolo facultativo".

18. Villarroel R. Limitaciones a la investigación científica en personas con "discapacidad psíquica o intelectual”. El paradigma de una “ciudadanía biológica”. En:”Impacto de la nueva normativa legal sobre la investigación científica en Chile" 8o Taller de Bioética organizado por el Comité Asesor de Bioética FONDECYT de CONICYT. Área de Comunicaciones CONICYT. Stgo. Dic 2013. Págs. 29-37. ISBN: 978-956-7524-18-1.

19. Díaz E. El acceso a la información de la ficha clínica. En: "Impacto de la nueva normativa legal sobre la investigación científica en Chile" $8^{\circ}$ Taller de Bioética organizado por el Comité Asesor de Bioética FONDECYT de CONICYT. Área de Comunicaciones CONICYT. Santiago, dic 2012. Págs. 49-78. ISBN: 978-956-7524-18-1.

20. Lavados M, Salas SP. Problemas éticos en los proyectos de investigación biomédica presentados al Comité de Ética de la Escuela de Medicina de la Pontificia Universidad Católica de Chile. Rev Med Chile 1997; 125 (9): 1011-8.

21. Téllez R. El rol de los Comités de Ética de la investigación en el nuevo marco legal. En "Impacto de la nueva normativa legal sobre la investigación científica en Chile" $8^{\circ}$ Taller de Bioética organizado por el Comité Asesor de Bioética FONDECYT de CONICYT. Área de Comunicaciones CONICYT. Stgo. Dic 2012. Págs. 39-46. ISBN: 978-956-7524-18-1. 